\title{
Quantum Drones Take Flight
}

\author{
A small prototype of a drone-based quantum network has successfully \\ relayed a quantum signal over a kilometer of free space.
}

By Michael Schirber

$\prod$ he airwaves are chock full of "classical" information from cell phones, radio stations, and Wi-Fi hubs, but one day those waves could be carrying quantum encrypted messages or data input for a quantum computer. A new experiment has used a pair of hovering drones to dole out quantum information to two ground stations separated by $1 \mathrm{~km}$ [1]. This demonstration could lead to a drone-based quantum network that could be positioned-and easily repositioned-over a city or rural area.

Quantum communication promises fully secure message sharing. For example, two users could exchange encrypted messages using "entangled" photons, pairs of particles with a unique quantum-mechanical relationship. For every pair, one photon would be sent to each of the users, who would be alerted to any eavesdropping by a loss of entanglement

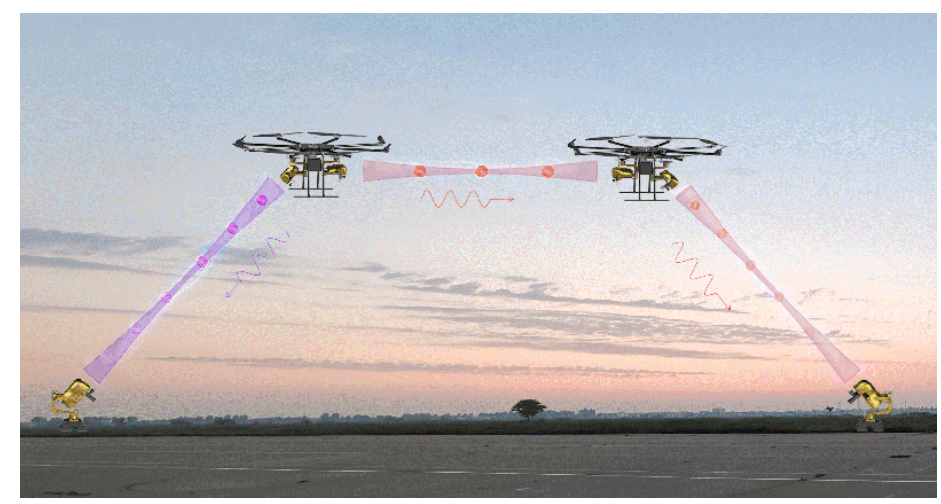

Droning on. A new quantum communication system consists of a drone (left) that generates entangled photon pairs and distributes them to ground stations. From each pair, one photon (purple beam) goes directly to the ground, while the other (pink beam) is relayed through the second drone (right).

Credit: X.-H. Tian, H.-Y. Liu, \& Z. Xie/Nanjing Univ.

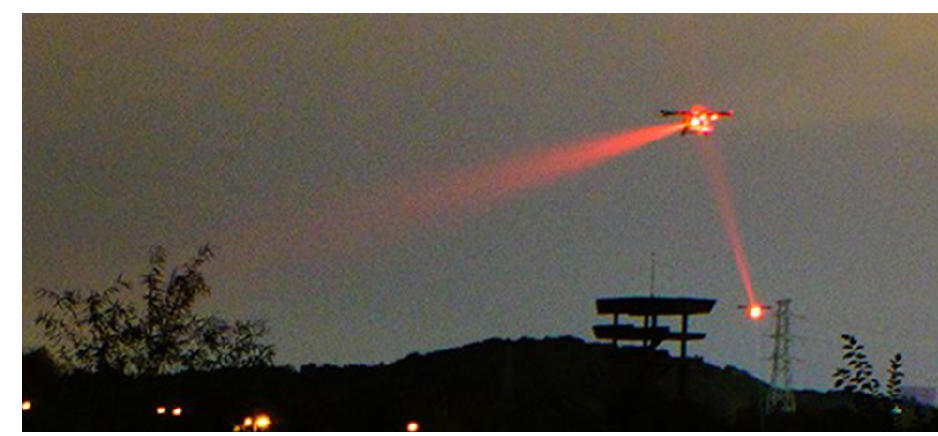

Target acquisition. The beams from one drone are visible over the testing area.

Credit: X.-H. Tian, H.-Y. Liu, \& Z. Xie/Nanjing Univ.

between the photons. One of the most common methods for sending such quantum encrypted messages relies on optical fibers (see Viewpoint: Record Distance for Quantum Cryptography). But in fibers, a large fraction of the photons scatter before reaching their destination. More photons can survive if quantum information is transmitted through the atmosphere, as in the quantum link established using a Chinese satellite in 2018 (see Focus: Intercontinental, Quantum-Encrypted Messaging and Video). However, satellites are expensive and difficult to adapt to changing demands on the ground.

Small drones carrying optical equipment could provide a flexible solution that could link multiple users in a quantum network. "Drones can be deployed for a mobile quantum connection at any given time and location when necessary," says Zhenda Xie from Nanjing University in China. Unlike a fixed tower, drones can also move around to avoid pollution or fog.

Several teams around the world have been working on drone-based systems. Early last year, Xie and colleagues 
reported a quantum link using a single octocopter-style drone [2]. The drone generated pairs of infrared photons whose polarization orientations were entangled. Using a high-speed tracking system, the drone directed one photon to a ground station labeled Alice and the other to a ground station labeled Bob. To collect the incoming photons, each station was equipped with a telescope having a $26-\mathrm{mm}$-wide aperture and a single-photon detector.

However, a major challenge to this form of optical communication comes from diffraction. As each photon propagates, its wave front spreads out, like the beam from a flashlight. If this spreading makes the wave front larger than the telescope aperture, the photon will have little chance of being collected. The team selected a short station-to-station distance of $200 \mathrm{~m}$ to ensure that diffraction effects were negligible.

To increase station separation, the team has now added a second drone that acts as a relay between the first drone and Bob. This drone collects photons from the first drone and collimates them through an optical fiber. This process reshapes the photon wave fronts-similar to what a focusing lens does-so that the photons have a higher chance of reaching Bob's telescope.

In a demonstration, the team positioned the two drones in between Alice and Bob, with drone-to-drone separation of 200 $\mathrm{m}$ and drone-to-station separation of $400 \mathrm{~m}$-giving a station-to-station distance of $1 \mathrm{~km}$. The Alice detector recorded about $25 \%$ of the photons sent its direction from the first drone, while the Bob detector recorded about $4 \%$ of the photons sent towards it.

The team performed a version of the so-called Bell inequality test by comparing the photon polarizations received at Alice and Bob. The results confirmed that the photons remained entangled, so the quantum information survived the trip. The team is now planning to enlarge the size of the network with multiple drones that could provide quantum links across a city, for example.

Georg Harder, a quantum engineer at the Paris-based company Veriqloud, has experience building photon-entanglement systems on large optical tables. "It made me smile when I read that the authors managed to put it all into a drone" he says. He adds that this demonstration opens up new options for quantum communication. "So far, quantum networks require either dedicated fiber networks or very expensive satellite links. The drones complement these existing systems."

One advantage of a drone system is that it can allow free-space communication between partners that do not have a direct line of sight, says Martin Bohmann, a quantum information specialist at the Austrian Academy of Sciences in Vienna. He points out that photon transmission losses need to be reduced to make a multidrone system competitive with other quantum network technologies, but he believes that such improvements are possible.

Michael Schirber is a Corresponding Editor for Physics based in Lyon, France.

\section{REFERENCES}

1. H.-Y. Liu et al., "Optical-relayed entanglement distribution using drones as mobile nodes," Phys. Rev. Lett. 126, 020503 (2021).

2. H.-Y. Liu et al., "Drone-based entanglement distribution towards mobile quantum networks," Natl. Sci. Rev. 7, 921 (2020). 